University of Wollongong

Research Online

Faculty of Engineering and Information

Faculty of Engineering and Information

Sciences - Papers: Part A

Sciences

$1-1-2013$

Quantitative model for tunable microstructure in magnetic FePt thin films by pulsed laser deposition

Igor A. Golovchanskiy

University of Wollongong, ig684@uowmail.edu.au

Sergey A. Fedoseev

University of Wollongong, sergey@uow.edu.au

Alexey V. Pan

University of Wollongong, pan@uow.edu.au

Follow this and additional works at: https://ro.uow.edu.au/eispapers

Part of the Engineering Commons, and the Science and Technology Studies Commons

Research Online is the open access institutional repository for the University of Wollongong. For further information contact the UOW Library: research-pubs@uow.edu.au 


\title{
Quantitative model for tunable microstructure in magnetic FePt thin films by pulsed laser deposition
}

\author{
Abstract \\ Pulsed laser deposition (PLD) is employed to fabricate FePt L10 thin films from elemental targets. \\ Dramatic structure variations are obtained by varying the laser frequency while keeping the thickness of \\ the films constant. A new theoretical model based on the mean field approach is proposed, which \\ quantitatively describes the structural changes obtained experimentally. The experiment and the model \\ exhibit the opposite growth development to the trend reported for the modulated flux in the literature. The \\ new model considers different growth rates in the lateral and transverse directions due to different \\ responses of the normal and tangential film surfaces being deposited to the incident flux and the \\ migration kinetics of adatoms and clusters. The quantitative results obtained confirm that the migration \\ kinetics and self-assembly can easily be controlled by the PLD frequency which is consistent with the \\ experiments. Magnetic properties of the films are shown to be extremely sensitive to the structure \\ variations allowing practical tunability. (C) 2013 IOP Publishing Ltd.
}

\section{Keywords}

thin, fept, magnetic, microstructure, tunable, model, deposition, quantitative, laser, pulsed, films

\section{Disciplines}

Engineering | Science and Technology Studies

\section{Publication Details}

Golovchanskiy, I. A., Fedoseev, S. A. \& Pan, A. V. (2013). Quantitative model for tunable microstructure in magnetic FePt thin films by pulsed laser deposition. Journal of Physics D: Applied Physics, 46 (21),

215502-1-215502-8. 


\title{
Quantitative model for tunable microstructure in magnetic FePt thin films by pulsed laser deposition
}

\author{
Igor A. Golovchanskiy, Sergey A. Fedoseev and Alexey V. Pan* \\ Institute for Superconducting and Electronic Materials, University of Wollongong, \\ Northfields Avenue, Wollongong, NSW 2522, Australia \\ *E-mail: pan@uow.edu.au
}

\begin{abstract}
Pulsed laser deposition (PLD) has been employed to fabricate FePt $L 1_{0}$ thin films from elemental targets. Dramatic structure variations have been obtained by varying the laser frequency while keeping the thickness of the films constant. A new theoretical model based on the mean field approach is proposed, which quantitatively describes the structural changes obtained experimentally. The experiment and the model exhibit the opposite growth development to the trend reported for the modulated flux in the literature. The new model considers different growth rates in lateral and transverse directions due to different responses of the normal and tangential film surfaces being deposited to the incident flux and the migration kinetics of adatoms and clusters. The quantitative results obtained confirm that the migration kinetics and self-assembly can easily be controlled by the PLD frequency which is consistent with the experiments. Magnetic properties of the films are shown to be extremely sensitive to the structure variations allowing practical tunability.
\end{abstract}

PACS numbers: 81.15.-z, 68.55.-a, 75.70.-i, 75.50.Vv

Published in J. Phys. D: Appl. Phys. 46 (2013) 215502 (8pp)

\section{Introduction}

A high degree of control is required for technology dealing with island-structured film fabrication to obtain desired properties and structures for film's optimal performance in applications. In physical vapour deposition (PVD) methods, to achieve a desired island structure, one conventionally manipulates with many parameters, including temperature, flux of material, resultant film thickness $[1,2,3]$ and deposition pulse frequency $[4,5,6,7]$ (in case of pulsed laser deposition). However, most of these parameters are interdependent, and their adjustment is not so straightforward (e.g. temperature, flux, thickness). In addition, the frequency in pulsed-based depositions influences the film structure in many different ways, whose mechanisms can be controversial and not always obeying available explanations $[4,5,6,7]$.

Most often, PVD FePt thin films are fabricated by magnetron sputtering. Its low growth rate and equiatomic ablation lead to superlattice formation with more 
equilibrium conditions than, for example, more rapid growth and different material delivery conditions of pulse laser deposition (PLD). However, magnetron sputtering may be considered as less technologically flexible for the growth control due to continuous material flux delivery and less practically viable due to lower film growth rates than PLD. The features of the advanced kinetics typical for PLD film growths, benefiting from high and chopped (pulsed) flux of ablated materials, have been well explored $[4,5,6,7]$. It has been shown that unlike chemical synthesis, the growth upon PLD process can be manipulated for both in-plane and out-of-plane directions nearly independently. However, the benefits of the predicted PLD growth have not been extensively employed for manufacturing of FePt films [8], most likely due to the expectations of obtaining poor film properties in comparatively low vacuum of typical PLD systems, as oppose to the high vacuum necessary for deposition of high purity (quality) metallic films.

The FePt PVD thin films with the $f c t$ crystal structure are promising candidates for various magnetic applications. The high magnetic anisotropy $\left(K_{u} \sim 7 \times 10^{6} \mathrm{~J} / \mathrm{m}^{3}\right)$ and high coercivity enable the size reduction of the thermally stable particles (or grains) down to a couple of nanometres $[9,10,11]$. Together with out-of-plane orientation of the easy magnetization axis and high corrosion resistance, these properties make FePt films with the desirable candidate for the perpendicular magnetic recording media with the recording density exceeding 1 TBit/in ${ }^{2}$ [12]. On the other hand, a very high coercivity (exceeding $10 \mathrm{~T}$ ) [1] and the corresponding high magnetic energy product open up new possibilities for the applications of FePt, such as microelectromechanical systems (MEMS) [13], ultra strong permanent magnets [11], or ferromagnetic-superconducting heterostructures [14].

In particular, smallest possible island size is required in recording media technology for high recording density $[9,15]$, in MEMS and permanent magnets for the highest coercive field possible $[1,2]$. In ferromagnetic-superconducting combinations certain FePt structures are desirable for optimal magnetic field compensation effect[14] (in somewhat similar fashion as soft ferromagnets can extend the range of Meissner effect in superconductors [16] and enhance their current carrying ability [17]).

Thickness reduction has been shown to be a common approach leading to small sized islands in PVD thin films $[1,2,3]$. However, excessive thickness reduction diminishes the read-back signal of recording media that should remain sufficiently high. On the other hand, the thickness decrease reduces the magnetic energy product of permanent magnets which is as crucial as high coercivity for these applications.

In this work, we demonstrate the advantages of pulsed laser deposition technique in tuning desirable structures and properties of the FePt epitaxial films, compared to other PVD systems. It can effectively be achieved by a simple control of PLD parameters for this binary compound, compared to a more complicated multielement compositions [18]. We propose and experimentally confirm the new behaviour of thin film growth kinetics, which can be easily tuned by laser frequency. This approach is likely to be suitable also for a range of similar materials requiring accurate growth control and particular surface architecture. 


\section{PLD of FePt thin films}

For magnetic recording purposes, it is preferred to grow FePt thin films on glass wafers [9]. However, for the epitaxial growth of the $f c t$ FePt films, it is better to use cubic (001) $\mathrm{MgO}$ or $\mathrm{SrTiO}_{3}$ (STO) single crystal substrates. Usually, $\mathrm{MgO}$ is used because the lattice mismatch between the $\mathrm{MgO}$ and FePt crystal structures is larger than that between STO and FePt, which leads to enhanced tensions at the interfaces [19]. The enhanced tensions provide a smaller size of islands during the PLD deposition and a better chemical ordering in the $f c t$ structure. However, we have employed STO substrates to ensure the reproducibility of the experiments, which cannot be guaranteed in $\mathrm{MgO}$ single crystals due to their ageing which requires additional treatment, such as cleaning by ion etching, before the deposition. Thus, the superior properties obtained in our FePt films can be further improved by using $\mathrm{MgO}$ substrates if high quality surface properties can be ensured.

In this work, the same PLD system used to grow high quality $\mathrm{YBa}_{2} \mathrm{Cu}_{3} \mathrm{O}_{7}$ thin films and multilayers has been employed [20]. However, for the FePt film deposition, the base pressure in the PLD chamber was typically held at $\leq 10^{-8}$ Torr. The pulsed laser deposition of the films has been carried out with the substrate temperature held at $800^{\circ} \mathrm{C}$, so that the vacuum deteriorated down to $10^{-7}-10^{-6}$ Torr due to thermal desorption from heater. The deposition temperature was chosen to ensure that the $L 1_{0}$ structure of the $\mathrm{FePt}$ alloy forming at $500^{\circ} \mathrm{C}[21,22,23]$ is complete $[2,8,13,19,24]$. The deposition process has been followed by annealing at the same temperature for half an hour.

The deposition was performed with $\mathrm{KrF}$ excimer laser (the wave-length of $248 \mathrm{~nm}$ ) by alternating elemental $4 \mathrm{~N}$ purity $\mathrm{Fe}$ and Pt targets. The laser fluency was kept at $\sim 3-4 \mathrm{~J} / \mathrm{cm}^{2}$, but taking into account optical path losses and laser beam homogenization, the fluency of $\sim 2 \mathrm{~J} / \mathrm{cm}^{2}$ is more realistic estimation. To facilitate nearly equiatomic composition in the FePt epitaxial superlattices (see, for example, $[25,26])$, the number of laser shots on the targets per one deposition period was 34 for Fe and 18 for Pt (taking into account different ablation [27, 28] and reevaporation properties for $\mathrm{Fe}$ and $\mathrm{Pt}$ ) with 12 periods in total. The composition of the films was verified by Energy Dispersive X-Ray Spectroscopy over the film surfaces. The deposition frequency was varied from $1 \mathrm{~Hz}$ to $6 \mathrm{~Hz}$, so that the resultant thickness of the films was approximately $20 \mathrm{~nm}$. The programmable target carousel in the PLD chamber was used to facilitate the non-interrupting deposition process.

\section{Tuning surface architecture}

Figs. 1 and 2 show respectively SEM and AFM images of the typical surface structures in the FePt thin films deposited at different laser frequencies. Generally, these structures are typical for the Volmer-Weber 3D island growth mode of metals on insulators [6, 29]. It is this growth mode which is dealt with in this work, unless specified otherwise. 

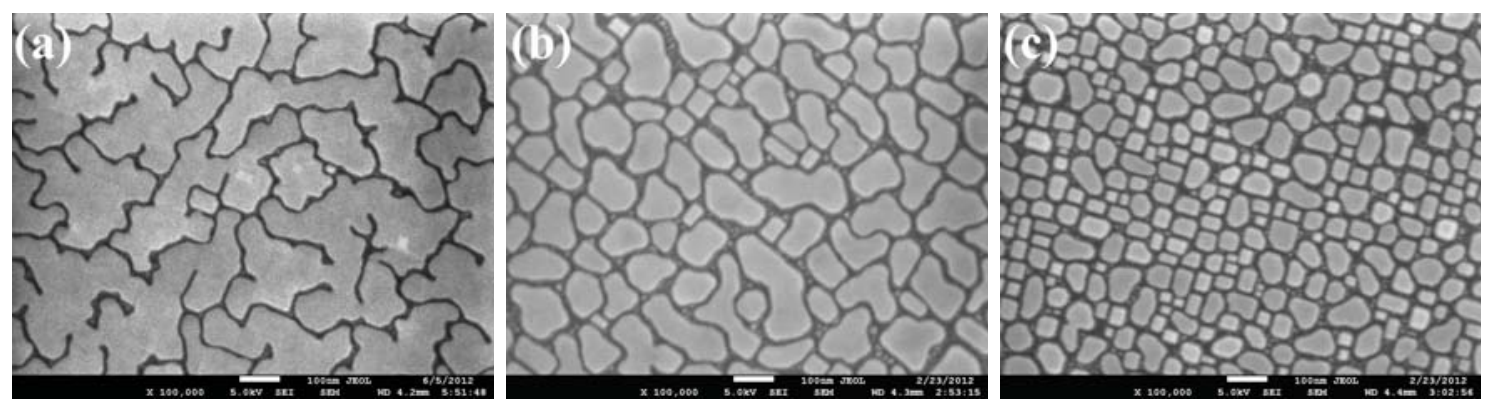

Figure 1. The surfaces of the FePt films deposited at $1 \mathrm{~Hz}$ (a), $2 \mathrm{~Hz}$ (b), and $6 \mathrm{~Hz}$ (c) obtained by scanning electron microscopy (SEM).

The epitaxial growth within the islands can be identified (i) in Figs. 1(b,c) from a clear rectangular shape translated from the substrate crystal lattice and characteristic orientations of some (in particularly smaller) islands along the crystallographic axes; and (ii) by the square-like shape of the hysteresis loops with strong coercive forces for $2 \mathrm{~Hz}$ and $6 \mathrm{~Hz}$ deposition frequencies for the out-of-plane magnetization measurements (Fig. 3), and narrow hysteresis with weak coercive fields and remanent magnetisation for the in-plane magnetisation measurements (not shown). This indicates high magnetic anisotropy typical for highly ordered $f c t$ FePt with the out-of-plane easy magnetisation axis [22].

According to the terminology established in Refs. [6, 7], the FePt film deposited at $1 \mathrm{~Hz}$ has undergone the percolation transition resulting from heavily amalgamating islands (filling holes in the film). On the other hand [Figure 1(c)], the films deposited at $6 \mathrm{~Hz}$ exhibit well-defined rectangular (if not square) island architecture with rather narrow size $(\sim 50 \mathrm{~nm}$ to $100 \mathrm{~nm})$ and shape distributions. The $2 \mathrm{~Hz}$ film [Figure 1(b)]demonstrates an intermediate case with a certain degree of islands coalescence and elongation (from $\sim 50 \mathrm{~nm}$ to $300 \mathrm{~nm}$ ), but no percolation. The island growth, coalescence, elongation, percolation and hole-filling are usual stages during the film growth process, which occur one after another as the thickness increases and more material is deposited on the substrate. The thickness of the film, at which any mentioned transition occurs, depends on the temperature of the substrate and the flux of the ablated material.

Note, this is the first time when all these growth stages are observed at the same temperature, flux and thickness by changing only the laser frequency (with the laser pulse duration kept constant). Moreover, our results confirming our theoretical prediction are at odds with existing results $[4,5,6,7]$. Obviously, this new rather simple control of the architecture in the PLD FePt films should provide not only their effective properties manipulation and tuning, but also technological advantages for practical applications with faster and more versatile depositions compared to magnetron sputtering or other deposition parameter control (such as temperature, vacuum, etc). Moreover, it is likely to be applicable for other similar type of films and applications. 

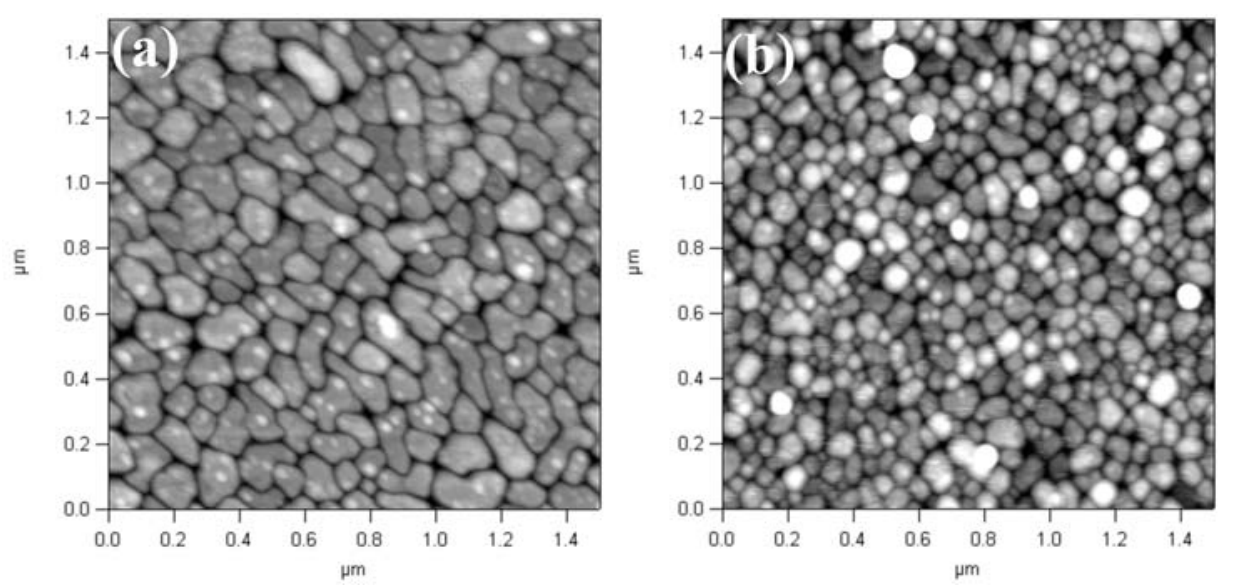

Figure 2. The surfaces of the FePt films deposited at $2 \mathrm{~Hz}$ (a) and $6 \mathrm{~Hz}$ (b) obtained by atomic force microscopy.

In Figure 2, the surface morphologies obtained by atomic force microscopy (AFM) of the films deposited at $2 \mathrm{~Hz}$ and $6 \mathrm{~Hz}$ are shown. Although the main film features observed with SEM and AFM are very similar, finer details appear to be different in these techniques. While AFM reveals some features $(<20 \mathrm{~nm})$ on the top of the islands (sub-island structure or droplets ablated from the target) which are best visible in Figure 2(a), these features are not observed by SEM at the resolution used. On the other hand, SEM shows some tiny islands of $<10 \mathrm{~nm}$ (or even clusters) in the channels between the major islands [best visible in Figure 1(b)], whereas these fine islands are unreachable for the AFM tip.

The studies of the early stages of the PLD growth have shown $[4,5]$ that the deposition process can be divided into three regimes, depending on the lifetime of adatoms $(\tau)$, the laser pulse duration $\left(\tau_{p}\right)$, and the PLD period $(1 / f)$. We are interested in the intermediate regime, i.e. $\tau_{p}<\tau<1 / f$, because our deposition satisfies this criterion. Indeed, we take our $f=1 \mathrm{~Hz}$ to $10 \mathrm{~Hz}$ and $\tau_{p} \sim 10 \mathrm{~ns}$ (for our eximer laser). While we can estimate $\tau$ from the assumption that in our process, with the substrate is heated to $800^{\circ} \mathrm{C}$ during the deposition, the lifetime of adatoms is mostly driven by strong re-evaporation (rather than diffusion) with the time scale given by [29]

$$
\tau_{e} \sim \nu^{-1} \exp \left(\frac{E_{a}}{k T_{\mathrm{sub}}}\right)
$$

where $E_{a}$ is the re-evaporation energy (a typical value is $\sim 1.6 \mathrm{eV}$ ) [30]), $k$ is the Boltzmann constant, $T_{\text {sub }}$ is the substrate temperature, and $\nu$ is the adatom reevaporation attempt frequency with a typical value being of $\sim 10^{11}-10^{13} \mathrm{~Hz}[29]$. It gives as $\tau_{e} \sim 0.005 \mathrm{sec}$, which falls within the limits of the regime considered. In this regime, the density of the islands has been shown $[4,5]$ to vary by $f$ with the assumption that the product $\tau_{p} f$ remains constant. In this case, the density would decrease if $f$ increases due to smaller amount of material ablated (as $\tau_{p}$ would decrease). This is valid at the beginning of the island nucleation, before the $15 \%$ coverage of the film surface is 


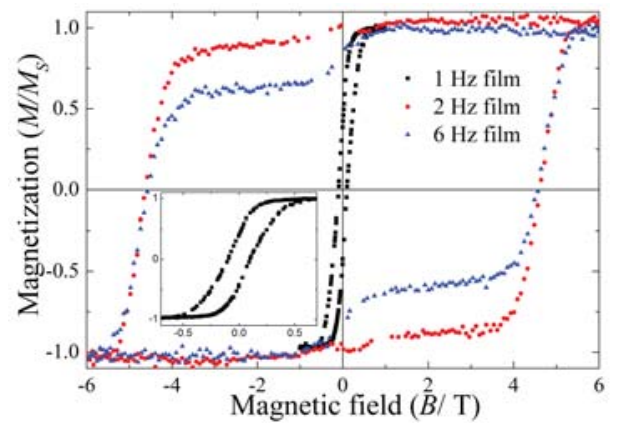

Figure 3. Hysteresis loops of the FePt films deposited at different frequencies with a field applied perpendicular to the surface of the films. The inset shows hysteresis loop of the film deposited at $1 \mathrm{~Hz}$.

reached and no significant impingement between islands occurs. In contrast, $\tau_{p}$ is not varied in our work. This would mean that the island concentration should not depend on $f$, whereas we observe a strong dependence on the PLD frequency.

The later stages of the PLD film growth are studied and modelled in Refs. [6, 7, 31] with the main processes being impingement, coalescence and elongation of the islands. The elongation transition is triggered as soon as the coalescence time scale $\tau \sim R^{4}$ (where $R$ is the radius of the island) becomes larger than the impingement time scale $[6,29,31]$. The latter is proportional to the lateral growth time scale of 3D growing islands. Therefore, if we still consider an increasing $f$, we should expect a decreasing time for impingements, which in turn (remembering that the number of the islands does not increase) leads to the elongation (increased size of the islands) and eventually to the percolation. We can see some general confirmation of this trend in Figs. 1 and 2. The larger islands in Figs. 1(b) and 2(a) are elongated whereas the smaller islands in Figs. 1(c) and 2(b) are separated and tend to coalesce. However, this trend observed has a major difference to the model described above. The density of the islands rises with the increasing frequency. Note, it is not a thermodynamically driven dependence since the deposition temperature and the annealing time have been kept identical for all the films.

Thus, we observe that the size of the islands is of the same order for different films, but distinctly larger for the film deposited at $2 \mathrm{~Hz}$ than for the film deposited at $6 \mathrm{~Hz}$ (Figs. 1 and 2). Taking into account these observations, we propose the following scenario with the central assumption being that the laser frequency affects the ratio of the out-of-plane to in-plane growth. At lower frequencies, if substrate surface coverage is significant (Fig. 5(c-f)), the islands tend to grow preferably in-plane, eventually experiencing impingements; whereas at higher frequencies they prefer to grow out-ofplane, remaining separated. In this scenario, the thickness would obviously be sensitive to the ratio changes, which is shown to be a critical factor in the film growth transitions $[2]$.

The properties of our FePt films appear to be easily tailored via their strong 


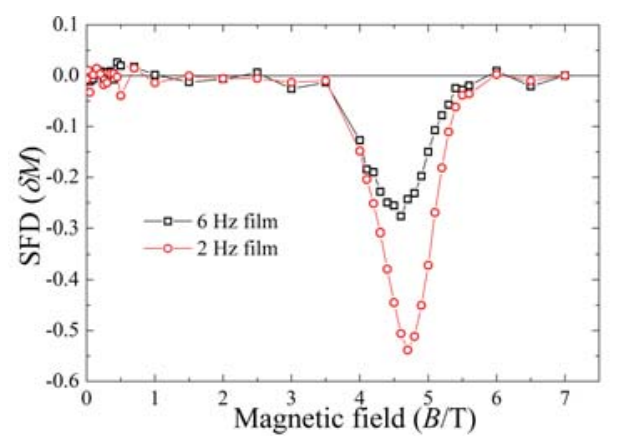

Figure 4. Swithching field distribution of the FePt films deposited at different frequencies.

dependence on the structure, which in turn can readily be controlled by varying the PLD frequency. Indeed, the film deposited at $1 \mathrm{~Hz}$ consists of the large interconnected percolative islands [Figure 1(a)] and demonstrates a narrow hysteresis loop with a small coercive field of $\sim 0.1 \mathrm{~T}$ (Figure 3), which is determined by the quasi-continuous surface structure. It creates negligible pinning for magnetic domain walls, which are rather freely move through the quasi-continuous film structure. The films deposited at higher frequencies exhibit qualitatively different surface patterns [Figure 1(b,c)] and correspondingly dramatic difference in the magnetic behaviour with very large coercive fields (Figure 3). The hysteresis loops measured in the magnetic field applied perpendicular to the surface of the films are typical for highly ordered $f c t$-FePt island structures with the easy magnetization axis being out-of-plane of the films. The hysteresis loops in Figure 3 for the films deposited at $f>1 \mathrm{~Hz}$ reveal a step-like feature around zero applied field. These steps were shown to be the soft magnetic contribution from poor chemical ordering [1] or/and as a result of pure Fe droplets incorporated in the films, which can be a by-product of the PLD process [13]. The $2 \mathrm{~Hz}$ film shows a much smaller step compared to the $6 \mathrm{~Hz}$ film probably due to the differences in the growth kinetics producing larger islands and more likely forming more ordered structure and composition across the $2 \mathrm{~Hz}$ film. Droplets of any kind have not been observed in our films by SEM. In general, there are several technological ways of removing these steps, such as the increase of the deposition temperature, annealing time, or laser energy. To emphasise the influence of the film structure on physical properties, we have obtained the so-called switching field distribution (SFD) for both films[32, 33]. The switching field distribution (Figure 4) shows how the relevant structures (islands or domains of a film) reverses the direction of its magnetization. At the coercive field $(\sim 4.6 \mathrm{~T})$ both films demonstrate strong dipolar interactions between islands. However, these interactions are by a factor of two smaller for the $6 \mathrm{~Hz}$ film than that for the $2 \mathrm{~Hz}$ film. A lower level of the interactions between the islands indicates that the structure obtained at $6 \mathrm{~Hz}$ is much more suitable for applications than the one obtained at $2 \mathrm{~Hz}$. It also again emphasizes the ease of the tunability of magnetic properties which can be achieved by varying the deposition frequency. 


\section{Quantitative growth model}

To check the scenario described above, we have employed the mean field theory $[5,29]$. Considering typical island geometry being rectangular at a stage with relatively high film coverage, we can divide all the surface of interest into three zones: (i) top of the islands, (ii) side of the islands, and (iii) the bare substrate between the islands. At this stage, we turn on our model deposition neglecting an opportunity for new island formation (restricting the largest possible cluster size by $n$ atoms), in particular taking into account the high deposition temperature facilitating high mobility of even large clusters [34, 35]. The main difference between these zones is determined by the deposition vector and the adatom/cluster absorption. Obviously, the sides of the islands (parallel to the deposition vector) obtain much less material for growth than the top of the islands. Hence, for the islands to grow in-plane adatoms/clusters should migrate from the top of the islands and from the substrate. In this case, the islands would have different rate of absorption for incoming material, whereas the mechanism of absorption is less important.

We can derive growth rate equations which depend on the densities of the adatoms $\left(\rho_{1}\right)$ and clusters $\left(\rho_{j}\right)$ on the top of the island as follows.

$$
\begin{aligned}
\frac{d \rho_{1}}{d t}= & F-F \sum_{i=1}^{n} i \rho_{i} \Omega^{2 / 3}-\frac{\rho_{1}}{\tau_{e}}-\sum_{i=1}^{n}\left(D_{1}+D_{i}\right) \rho_{1} \rho_{i}- \\
& -\frac{D_{1} \rho_{1}}{L^{2}}+2 \frac{D_{1}^{\text {side }} \rho_{1}^{\text {side }}}{L d} \\
\frac{d \rho_{j}}{d t}= & F(j-1) \rho_{j-1} \Omega^{2 / 3}+\frac{1}{2} \sum_{i=1}^{j-1}\left(D_{i}+D_{j-1}\right) \rho_{i} \rho_{j-i}- \\
& -\sum_{i=1}^{n}\left(D_{i}+D_{j}\right) \rho_{i} \rho_{j}-\frac{D_{j} \rho_{j}}{L^{2}}+2 \frac{D_{j}^{\text {side }} \rho_{j}^{\text {side }}}{L d}
\end{aligned}
$$

where the diffusion coefficient of a cluster with $i$ atoms is

$$
D_{i}=i^{-\alpha} \nu a^{2} \exp \left(-\frac{E_{d}}{k T_{\text {sub }}}\right)
$$

with $\alpha=1.5$ to 1.75 for clusters on an island and $\alpha=1.3$ for the clusters on a substrate $[34,35])$. In these equations, $F$ is the flux of adatoms per pulse, $\Omega$ is the atomic volume, $\rho_{i}$ is the number of clusters with $i$ atoms per unit area, $n$ is the critical size of mobile cluster (clusters with more than $n$ atoms are adsorbed by islands), $L$ is the lateral size of an island, and $d_{f}$ is the thickness of the film (the height of an island), $a$ - lattice parameter.

In the first term of Equation (2), we presume that all other adatom contributions are negligible compared to the incident flux. The second term represents the loss of adatoms by direct incident flux and their insertion into different size clusters. These adatoms (partially) re-emerge in size $j$ clusters after direct impingement with $(j-1)$ clusters in the first term of Equation (3) (and the second term in Equation 6). The third term in Equation (2) takes into account the evaporation of adatoms. The fourth term represents 
the loss of adatoms by coalescence with other clusters, which is similar to the third term in Equation (3) for clusters rather than adatoms. The fifth term in Equation (2) and the forth term in Equation (3) are responsible for the loss of adatoms/clusters by their migration from the top of islands to their sides. The last term in Eqs. (2) and (3) considers the reverse migration of adatoms/clusters from islands sides to their tops. The last two terms in both equations enable the in-plane island growth because material is not deposited on side of the island directly. The second term in Equation (3) emerges due to coalescence of two arbitrary clusters into size $j$ clusters. The island disintegration (breakage) is not taken into account (irreversible aggregation limit).

Similar rate equations to Eqs. (2) and (3) must be considered for the free surface of the substrate and for the sides of the islands. All six obtained equations are interconnected through terms similar to the last two terms in Eqs. (2) and (3), which describe possible migrations of adatoms shown in Figure5(e) by the arrows. However, in the two equations for the side of the islands there are obviously no terms responsible for the deposition of adatoms from the incident flux.

Finally, we consider the rate equations for the in-plane growth

$$
\frac{d L}{d t}=\frac{1}{2} \sum_{i=1}^{n} \sum_{j=n+1-i}^{n}\left(D_{i}^{\text {side }}+D_{j}^{\text {side }}\right) \rho_{i}^{\text {side }} \rho_{j}^{\text {side }}(i+j) \Omega
$$

and the out-of-plane growth

$$
\begin{aligned}
\frac{d d_{f}}{d t}= & \frac{1}{2} \sum_{i=1}^{n} \sum_{j=n+1-i}^{n}\left(D_{i}^{\mathrm{top}}+D_{j}^{\mathrm{top}}\right) \rho_{i}^{\mathrm{top}} \rho_{j}^{\mathrm{top}}(i+j) \Omega+ \\
& +F \Omega^{2 / 3} n \rho_{n}^{\mathrm{top}} \Omega
\end{aligned}
$$

which are computed simultaneously with the six rate equations for the atom/claster density. The details of this solution will be published elsewhere.

Figure 6 shows the ratio of the in-plane to out-of-plane growth rate dependence on the PLD frequency and different surface coverage obtained from Eqs. (5) and (6). Initial conditions for the computations were deduced from the film structures shown in Figs. 1 and 2 and experimental details. Islands dimensions ratio and density are kept constant independent of surface coverage. A model film with $75 \%$ coverage consists of rectangular islands having the size of $125 \times 125$ unit cells in-plane and 90 cells outof-plane with 20 unit cells gap between the neighbouring islands. These parameters of the modelled islands match with the size of the smallest islands of $\sim 40 \mathrm{~nm}$ inplane and $\sim 20 \mathrm{~nm}$ out-of-plane in Figs. 1(c) and 2(b), and correspond to deposition stage in Fig.5(e,f). A model film with $40 \%$ coverage consists of rectangular islands having the size of $90 \times 90$ unit cells in-plane and 65 cells out-of-plane with 55 unit cells gap between the neighbouring islands. It corresponds to the earlier deposition stage exhibited in Fig. 5(c,d). For each model film the initial density of adatoms/clusters was zero. Experimentally, it can be attributed to the deposition interrupted by switching targets from $\mathrm{Fe}$ to $\mathrm{Pt}$ at a certain surface coverage. This interruption would allow all adatom/cluster migration and adsorption processes to settle. The initial stage of the 

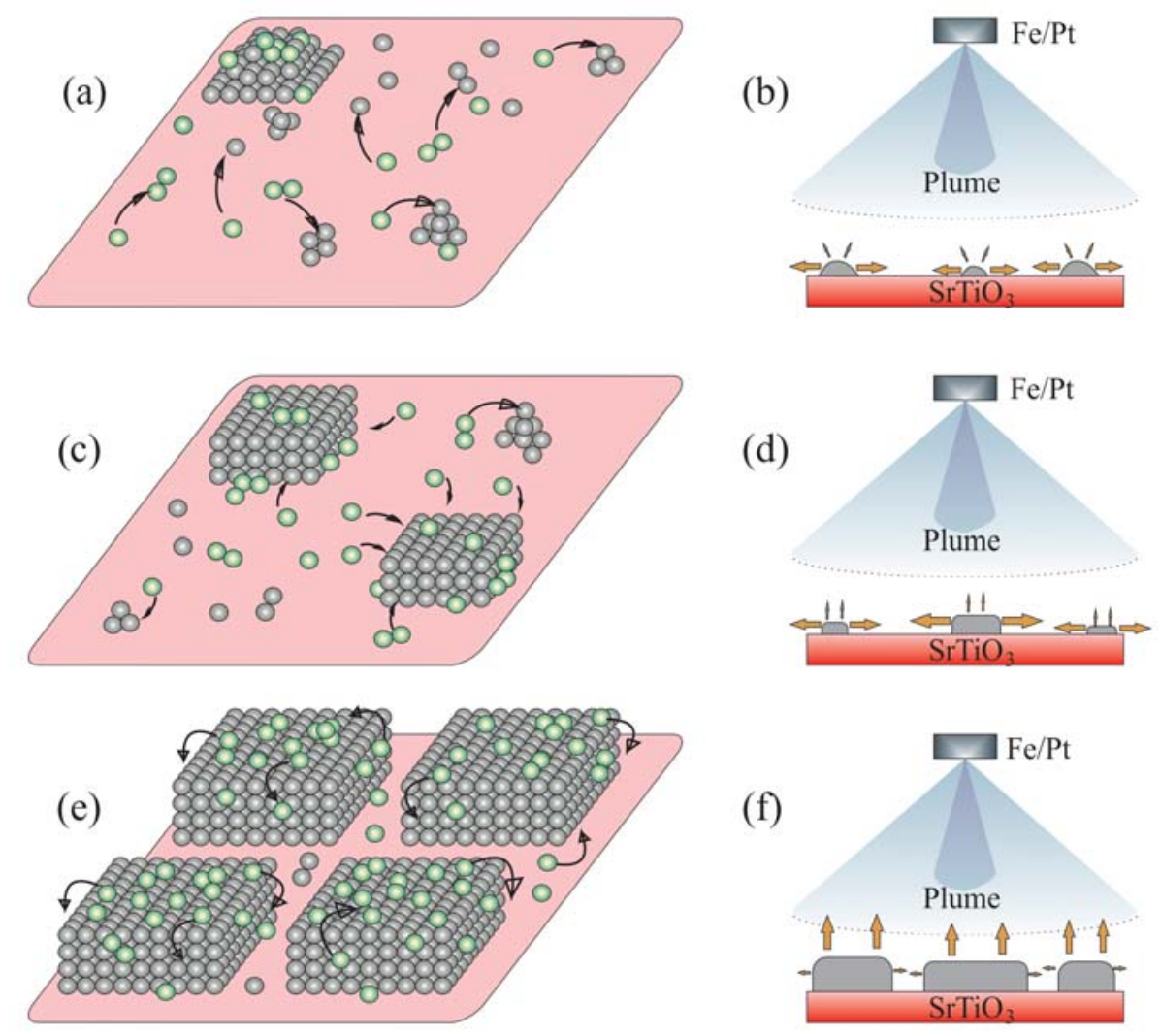

Figure 5. The schematic stages of the growth model proposed: (a,b) the very initial stage of island nucleation after just a few laser shots with insignificant substrate coverage with mostly $2 \mathrm{D}$ in-plane growth, which is not considered in our computations; $(\mathrm{c}, \mathrm{d})$ the intermediate stage with a low/medium coverage by a number of small islands still growing mostly in-plane; $(e, f)$ the late stage with a significant coverage of the substrate by the rectangular islands growing mostly out-of-plane, while the in-plane growth occurs as a result of adatom/cluster migration to the sides of the islands [the arrows in (e)]. Freshly deposited (hot) adatoms are shown in green, while the settled adatoms are depicted in grey $(\mathrm{a}, \mathrm{c}, \mathrm{e})$.

deposition (Fig. 5a,b) reflecting island nucleation is not considered in our computation. This is because these two stages of nucleation and then growth rely on respectively different growth and nucleation physical processes involved.

The flux $F=0.1$ atoms per laser pulse per square cell with 30 nanosecond pulse duration. The largest mobile cluster ( $n=50$ atoms) is assumed to travel the maximum distance comparable to the lateral dimension of the largest island over the duration of the deposition.

The computation has been done over the deposition frequency range from 1 to $100 \mathrm{~Hz}$. This range covers not only frequencies used for deposition in this work, but also partially covers frequencies of existing industrial high-rate pulsed laser deposition systems [36]).

The ratio of the growth rates obviously evolves with the number of shots (laser 

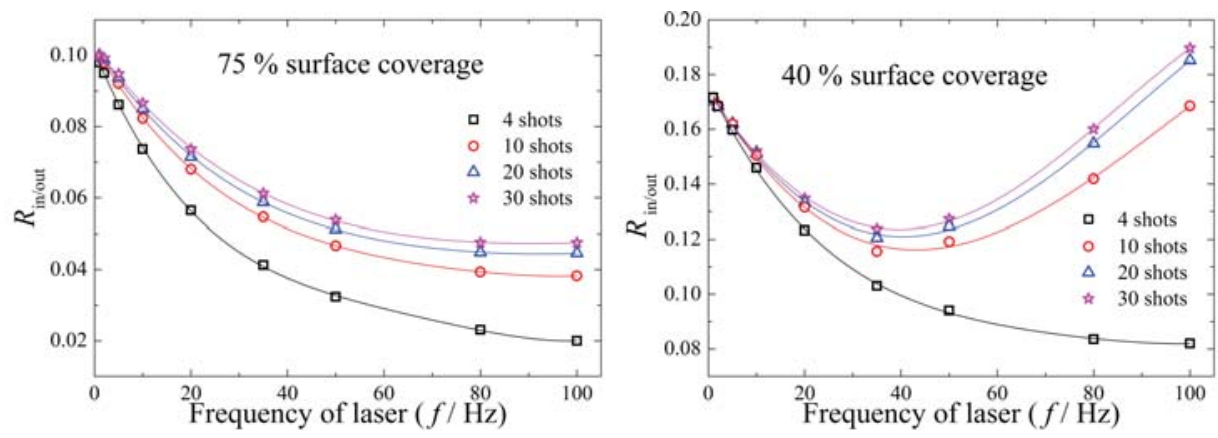

Figure 6. The ratio of the in-plane to out-of-plane growth rate dependence $\left(R_{\text {in/out }}\right)$ on the PLD frequency at different surface coverage calculated from the growth rate equations Eqs. (5) and (6). The lines are guides to an eye.

pulses). At low frequencies, the ratio is only marginally larger upon increasing the number of shots (Figure 6) for any surface coverage. Obviously, insufficient material is delivered to the substrate at low frequencies, hence the steady growth state does not significantly differ from just a few shots a large number of shots. At higher frequencies, the difference in the ratio notably increases with increasing number of shots. In this case, due to the large amount of delivered material more shots is required to reach the steady state. The in-plane growth is boosted by the amount of the delivered material, and the ratio can even reverse its trend from decreasing with increasing frequency (after 4 shots) to increasing with increasing frequency (after 10 shots) for the case of $40 \%$ surface coverage. This boost in the in-plane growth rate is due to a higher concentration of clusters on the sides of islands with increasing number of shots. In the case of the low surface coverage, the additional boosting factor is accumulation of clusters on the free surface of substrate, which is so substantial that enables the reverse in the ratio trend.

After $>20$ shots, the growth ratio reaches its certain equilibrium and does not change significantly, indicating that the steady growth state is reached over the entire frequency range considered. It is this state, which is normally fulfilled during the uninterrupted PLD process.

Note, the consideration above is applicable to the FePt film deposition, which requires the switching between $\mathrm{Fe}$ and $\mathrm{Pt}$ targets with the number of shots (34 for $\mathrm{Fe}$ and 18 for Pt) being in the range considered in the model. Indeed, every interruption required for the switching the target is assumed to facilitate the initial conditions of the growth, which are adopted for the model calculation.

In general, the ratio in Figure 6 is always below unity, this means that in this island geometry the growth is faster out-of-plane than in-plane. At the significant $(75 \%)$ substrate coverage [Figure 5(e,f)], the in-plane growth becomes even slower, so that slower depositions (low frequencies) would promote the in-plane growth, while faster depositions (higher frequencies) would result in taller island-like architecture (which is likely to eventually coalescence with a rough continuous surface morphology). Within the experimental deposition frequency range, the ratio of the in-plane to the out-of- 
plane island growth rate drops by $\sim 20 \%$ [Fig. 6(a)] if the frequency of laser deposition increases from $1 \mathrm{~Hz}$ to $6 \mathrm{~Hz}$.

If the surface coverage is rather low (40\%), the ratio decreases with increasing frequency until $\simeq 35 \mathrm{~Hz}$ and then increases at $f>40 \mathrm{~Hz}$. In the experimental frequency range $(1 \mathrm{~Hz}$ to $6 \mathrm{~Hz})$, the ratio of the in-plane to the out-of-plane island growth rate decreases by $\sim 5 \%$ [Fig. 6(b)]. At low deposition frequencies re-evaporation plays an important role decreasing adatom concentration. At this relatively low concentration, the major factor of growth kinetics is insensitivity of the sides of the islands to incident flux (the same mechanism as at high coverage). At high deposition frequency, the adatoms are mostly adsorbed by clusters and do not re-evaporate. In this case, the growth kinetics is controlled by the ratio of the island surface to free substrate surface. As soon as the number of shots is increased above a certain threshold the in-plane growth is dramatically accelerated at $f>40 \mathrm{~Hz}$, and the ratio starts increasing.

First of all, these results prove our assumption made on the basis of our experiment that the deposition frequency effectively affects the ratio of the in-plane to out-of-plane growth. Secondly, Fig. 6 demonstrates nontrivial behaviour of the ratio with frequency at different surface coverage. This result can be applied to any PVD system for the particular conditions considered in this work, while Eqs. (2), (3), (5) and (6) can be used to model architectures at a broad range of conditions.

\section{Conclusion}

In this work, FePt thin films have been fabricated by pulsed laser deposition. The structure of the films has effectively been controlled by the PLD frequency variation. Dramatic improvement (from the point of view of magnetic properties) of the in-plane film structure has been achieved for the films with a constant thickness by increasing the frequency. These structural changes contradict to the growth patterns and their models reported in the literature. We have proposed a new model to explain this behaviour. The new model considers the specific geometry of the film islands: for a substantial film coverage, the islands are considered to have rectangular cross-sections with identifiable out-of-plane oriented sidewalls parallel to the incident ablated material. This governs a slower in-plane growth compared to the out-of-plane growth. The model proposed provides quantitative agreement with the experimental results. The in-plane growth rate has been shown to decrease with increasing frequency, leading to the smaller individual islands at a certain thickness. Obviously, by increasing the laser frequency islands are prevented from impingement for certain thicknesses with the neighbouring islands proportionally to the slower in-plane growth rate.

The effective manipulation of the structural changes and its quantitative description offer a promising tool in tuning thin film physical properties important for applications. By the 6-fold variation of the frequency (from $1 \mathrm{~Hz}$ to $6 \mathrm{~Hz}$ ), we obtained the 20-fold variation in the coercive field (from $0.2 \mathrm{~T}$ to $4.6 \mathrm{~T}$ at room temperature, respectively) in the FePt thin film in spite of the relatively low vacuum used in our deposition chamber. 
There are a number of ways to further improve structure and properties of our films. First of all, the deposition of FePt films on the MgO substrates (instead of STO) should decrease typical size of island. Obtaining higher vacuum during PLD should even further increase the coercive field for high-performance films. Moreover, the deposition density of the islands at early deposition stages is proportional to the ratio of the material flux to its diffusion $\propto(F / D)^{\chi}[5]$, both can be controlled by the deposition parameters. Hence, increasing the island density at the early stages and preventing them from impingement and coalescence at later stages can significantly improve the island film performance for various applications.

\section{Acknowledgment}

This work is supported by the Australian Research Council.

\section{References}

[1] T. Shima, K. Takanashi, Y. K. Takahashi, K. Hono, Appl. Phys. Lett. 85, 2571 (2004)

[2] T. Shima, K. Takanashi, Y. K. Takahashi, K. Hono, Appl. Phys. Lett. 81, 1050 (2002)

[3] A. A. Baski, H. Fuchs, Surface Science 313, 275-288 (1994)

[4] P. Jensen, B. Niemeyer, Surf. Sci. 384, L823-L827 (1997)

[5] N. Combe, P. Jensen, Phys. Rev. B 57, 15553 (1998)

[6] J. M. Warrender, M. J. Aziz, Phys. Rev. B 76, 045414 (2007)

[7] M. J. Aziz, Appl. Phys. A 93, 579 (2008)

[8] S. Fahler, U. Hannemann, M. Weisheit, V. Neu, S. Melcher, S. Leinert, S. C. Wimbush, A. Singh, A. Kwon, B. Holzapfel, L. Schultz, Appl. Phys. A 79, 1529-1531 (2004)

[9] A. Moser, K. Takano, D. T. Margulies, M. Albrecht, Y. Sonobe, Y. Ikeda, Sh. Sun, E. E. Fullerton, J. Phys. D: Appl. Phys. 35, R157-R167 (2002)

[10] D. Weller, A. Moser, L. Folks, M. E. Best, W. Lee, M. F. Toney, M. Schwickert, J. Thiele, M. F. Doerner, IEEE Trans. Magn. 36, 10 (2000)

[11] S. D. Bader, Reviews of Modern Physics 78, 1 (2006)

[12] M.H. Kryder, E.C. Gage, T.W. McDaniel, W.A. Challener, R.E. Rottmayer, G. Ju, Y.-T. Hsia, M. Fatih Erden, Proceedings of the IEEE 96, 1811 (2008); Seagate demonstration in The Magnetic Recording Conference, USA, 2012

[13] S. Fahler, V. Neu, M. Weisheit, U. Hannemann, S. Leinert, A. Singh, A. Kwon, S. Melcher, B. Holzapfel, L. Schultz in (18th Workshop on High Performance Magnets and their Applications, Annecy, France, 2004)

[14] S. Haindl, M. Weisheit, T. Thersleff, L. Schultz, B. Holzapfel, Supercond. Sci. Technol. 21, 045017 (2008)

[15] H. Zhou, H. N. Bertram, IEEE Trans. Magn. 35, 2712 (1999)

[16] A. V. Pan, S. Zhou, H. Liu, S. Dou, Supercond. Sci. Technol. 16, L33 (2003)

[17] A. V. Pan, S. Dou, J. Appl. Phys. 96, 1146 (2004)

[18] S. Pysarenko, A. V. Pan, S. X. Dou, IEEE Trans. Appl. Supercond. 21, 3179 (2011)

[19] M. Weisheit, L. Schultz, S. Fahler, J. Appl Phys. 95, 7489 (2004)

[20] A. V. Pan, S. Pysarenko, S. X. Dou, Appl. Phys. Lett. 88, 232506 (2006)

[21] R. F. C. Farrow, D. Weller, R. F. Marks, M. F. Toney, S. Hom, G. R. Harp, A. Cebollada, Appl. Phys. Lett. 69, 1166 (1996)

[22] Y. Endo, N. Kikuchi, O. Kitakami, Y. Shimada, J. Appl Phys. 89, 7065 (2001) 
J. Phys. D: Appl. Phys. 46 (2013) 215502 (8pp)

[23] T. Suzuki,K. Harada, N. Honda, K. Ouchi, Journal of Magnetism and Magnetic Materials 193, $85(1999)$

[24] X. H. Li, B. T. Liu, W. Li, H. Y. Sun, D. Q. Wu, X. Y. Zhang, J. Appl. Phys. 101, 093911 (2007)

[25] M. H. Hong, K. Hono, M. Watanabe, J. Appl. Phys. 84, 4403 (1998)

[26] M. Weisheit, L. Schultz, S. Fahler, Journal of Magnetism and Magnetic Materials 290-291, 570$572(2005)$

[27] Y. Hiroshima, T. Ishiguro, I. Urata, H. Makita, H. Ohta, M. Tohogi, Y. Ichinose, J. Appl. Phys. 79, 3572 (1996)

[28] S. Amoruso, Appl. Phys. A 69, 323-332 (1999)

[29] J.A. Vanables, G.D.T. Spiller, M. Hanbucker, Rep. Prog. Phys. 47, 399 (1984)

[30] P. Stolze, J. Phys. Condens. Matter 6, 9495 (1994)

[31] G. Jeffers, M.A. Dubson, P.M. Duxbury, J. Appl. Phys. 75, 5016 (1994)

[32] H. Zeng, Sh. Sun, T. S. Vedantam, J. P. Liu, Z.-R. Dai, Z.-L. Wang, Appl. Phys. Lett. 80, 2583 (2002)

[33] P.E. Kelly, K. O’Grady, P.I. Mayo, R.W. Chantrell, IEEE Trans. Magn. 25, 3881 (1989)

[34] J.-M. Wen, S.-L. Chang, J. W. Burnett, J. W. Evans, P. A. Thiel, Phys. Rev. Lett. 73, 2591 (1994)

[35] P. Deltour, J.L. Barrat, P. Jensen, Phys. Rev. Lett. 78, 4597 (1997)

[36] A. Usoskin and H.C. Freyhardt, MRS Bulletin 29, 583 (2004) 